\title{
The distance of M 33 and the stellar population in its outskirts
}

\author{
S. Galleti ${ }^{1,2}$, M. Bellazzini ${ }^{1}$, and F. R. Ferraro ${ }^{2}$ \\ 1 INAF - Osservatorio Astronomico di Bologna, via Ranzani 1, 40127 Bologna, Italy \\ e-mail: silvia.galleti2@unibo.it; michele.bellazzini@bo.astro.it \\ 2 Dipartimento di Astronomia, Università di Bologna, via Ranzani 1, 40127 Bologna, Italy \\ e-mail: francesco.ferraro3@unibo.it
}

Received 22 March 2004 / Accepted 10 May 2004

\begin{abstract}
We present deep $V, I$ photometry of two $9.4^{\prime} \times 9.4^{\prime}$ field in the outer regions of the M 33 galaxy. We obtain a robust detection of the luminosity of the Red Giant Branch Tip $\left(I^{\mathrm{TRGB}}=20.72 \pm 0.08\right)$ from which we derived a new estimate of the distance modulus of M 33, $(m-M)_{0}=24.64 \pm 0.15$, corresponding to a distance $D=847 \pm 60 \mathrm{kpc}$. By comparison of the color and magnitude of the observed Red Giant Branch stars with ridge lines of template globular clusters we obtained the photometric metallicity distribution of the considered fields in three different metallicity scales. The derived metallicity distributions are very similar over a range of distances from the galactic center $10^{\prime} \leq R \leq 33^{\prime}$, and are characterized by a well defined peak at $[M / \mathrm{H}] \simeq-0.7([\mathrm{Fe} / \mathrm{H}] \simeq-1.0$, in the Zinn \& West scale) and a weak metal-poor tail reaching $[M / \mathrm{H}] \lessgtr-2.0$. Our observations demonstrate that Red Giant Branch and Asymptotic Giant Branch stars have a radial distribution that is much more extended than the young MS stars associated with the star-forming disc.
\end{abstract}

Key words. galaxies: individual: M 33 - galaxies: distances and redshifts

\section{Introduction}

${ }^{1}$ The accurate determination of the distance to Local Group galaxies is critical for the establishment of a reliable extragalactic distance scale. In particular, local late-type galaxies as the Large Magellanic Cloud, M 31 and M 33 are the sites of choice for the calibration of several secondary distance indicators.

The Sc II-III spiral M 33 (NGC 598 or Triangulum galaxy) is the third-brightest member of the Local Group (see Van den Bergh 2000, for a review). In spite of that, while Cepheid variables were discovered as early as 1920 in this galaxy (Hubble 1926), reliable distance estimates have become available only after 1980 (Sandage \& Carlson 1983; Christian $\&$ Schommer 1987) and differences of the order of $\sim 0.3 \mathrm{mag}$ can be found even among the most recent estimates of the M 33 distance modulus (see McConnachie et al. 2004, and references therein).

Concerning the stellar populations of M 33, most of the studies have centered their attention on the massive (young) stars populating the disc (see, for example Van den Bergh 1991, 2000; Urbaneja et al. 2002, and references therein), and/or on the innermost regions of the galaxy (Mighell \& Rich 1995;

${ }^{1}$ Based on observations made with the Italian Telescopio Nazionale Galileo (TNG) operated on the island of La Palma by the Centro Galileo Galilei of the INAF (Istituto Nazionale di Astrofisica) at the Spanish Observatorio del Roque de los Muchachos of the Instituto de Astrofisica de Canarias.
Stephens \& Frogel 2002). On the other hand, only a few authors have provided some insight into the outer regions and the (possible) halo population (see Mould \& Kristian 1986; Wilson et al. 1990; Davidge 2003, and the HST-WFPC2 study by Kim et al. 2002).

Here we present $V$ and $I$ photometry (reaching $V \sim 24.5$ ) of two fields located at $\sim 15^{\prime}$ and $\sim 28^{\prime}$ from the center of M 33, to the North-West of the main body of the galaxy, approximately in the direction of the minor axis (see Fig. 1). The Tip of the Red Giant Branch (TRGB) is cleanly detected with our data and we use it to derive a robust estimate of the distance modulus of M 33 adopting the RR Lyrae-independent calibration provided by Bellazzini et al. (2001), Bellazzini et al. (2004). This is the main aim of the present study which is part of a large programme devoted to the determination of homogeneous distances for most of the galaxies of the Local Group (see Bellazzini et al. 2002). The metallicity distribution of the RGB stars in the considered fields is also obtained from photometric estimates, following the method by Bellazzini et al. (2003). Shortly after this paper was submitted, a preprint was posted (Tiede et al. 2004, hereafter T04) presenting the analysis of a $6.8^{\prime} \times 6.8^{\prime}$ field located in the South-Eastern region of M 33 at $\sim 20^{\prime}$ from its center. The analysis is very similar to that performed here and the results are in excellent agreement, as we will show below.

The structure of the paper is as follows: in Sect. 2 we describe the observational material, the data reduction process and the photometric calibration; in Sect. 3 we present the 


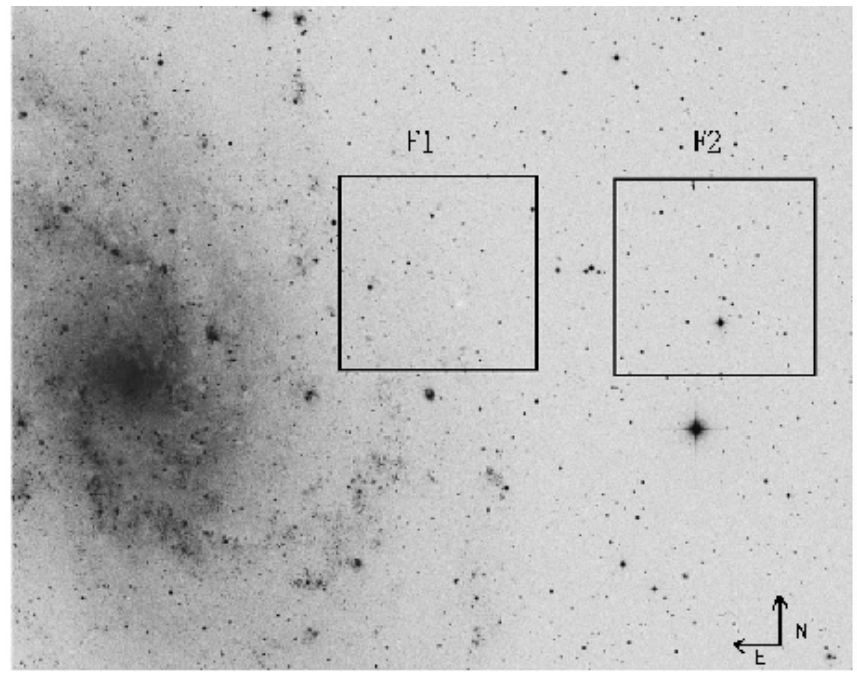

Fig. 1. The position of the observed fields over-plotted on a DSS-II image of M 33. The dimension of the image is $40^{\prime} \times 33^{\prime}$.

color-magnitude diagrams (CDMs), our TRGB estimate of the distance modulus, and the (photometric) metallicity distributions. The stellar content of the considered fields is also briefly discussed. A brief summary and the main conclusions are reported in Sect. 4.

\section{Observations and data reduction}

\subsection{Observations}

The observations were carried out on September 7 and 8, 2002, with the imager/spectrograph DoLoRes at the $3.52 \mathrm{~m}$ Italian telescope Telescopio Nazionale Galileo (TNG) in La Palma (Canary Island, Spain). DoLoRes is equipped with a $2048 \times$ 2048 pixel thinned and back-illuminated Loral CCD array with a total field of view of $9.4 \times 9 ! 4$, and a scale of $0.275^{\prime \prime} / \mathrm{px}$. The seeing was remarkably good and stable, ranging between $0.8^{\prime \prime}$ and $1.1^{\prime \prime} F W H M$ for all the scientific images.

Two fields were imaged along the minor axes in $V$ and $I$ filters. The locations of the fields are shown in Fig. 1 and listed in Table 1 along with the date, the filter and the exposure time of all the scientific exposures. A total of $7 \mathrm{~V}$ and $6 I 600 \mathrm{~s}$ exposures have been acquired for the inner field (Field 1 , hereafter F1) and $8 \mathrm{~V}$ and $6 I 600 \mathrm{~s}$ for the outer field (Field 2, hereafter F2). The distance of the field center from the center of the galaxy is $\simeq 15^{\prime}$ for $\mathrm{F} 1$ and $\simeq 28^{\prime}$ for $\mathrm{F} 2$.

\subsection{Data analysis}

All the images were corrected for bias and flat-field using standard IRAF procedures. The relative photometry was carried out with the PSF-fitting code DoPhot (Schechter et al. 1993). Each frame was independently reduced. A $3 \sigma$ threshold above the background noise was adopted for the search for sources on the frames and the spatial variations of the PSF were modeled with a quadratic polynomial function. Only the sources classified as stars by the code were retained. All the $V$ and $I$ catalogues of a given field were reported to the (instrumental) photometric
Table 1. Log of the observations.

\begin{tabular}{l|rrccc}
\hline \hline Field & RA (J2000) & Dec (J2000) & Date & Filt. & Exp T(s) \\
\hline F1 & 013244.85 & +304502.5 & Sept., 7, 02 & $V$ & $3 \times 600$ \\
& & & Sept., 7, 02 & $I$ & $3 \times 600$ \\
& & & Sept., 8, 02 & $V$ & $4 \times 600$ \\
& & & Sept., 8, 02 & $I$ & $3 \times 600$ \\
F2 & \multirow{2}{*}{013144.97} & +304500.0 & Sept., 7, 02 & $V$ & $5 \times 600$ \\
& & & Sept., 7, 02 & $I$ & $4 \times 600$ \\
& & & Sept., 8, 02 & $V$ & $3 \times 600$ \\
& & & Sept., 8, 02 & $I$ & $2 \times 600$ \\
\hline
\end{tabular}

system of the best-seeing image acquired under photometric conditions. The catalogues were then cross-correlated, the magnitudes were averaged and the standard deviation adopted as the photometric uncertainty of the individual stars. In the final catalogue of instrumental magnitudes and positions we have retained only the sources that have at least three valid measures of the magnitude for each passband (e.g. at least $3 \mathrm{~V}$ and $3 I$ ). Moreover, all the stars with an associated error (either in $V$ or $I$ magnitude) larger than three times the average uncertainty at their magnitude level were excluded from the sample. The final F1 and F2 catalogues contains 26399 and 1937 sources, respectively. Accurate aperture corrections have been obtained for each field on a few tens of bright and isolated stars.

The stellar crowding is quite low in the considered fields that sample external low-surface brightness regions of the galaxy. Even in F1 the average stellar density is as low as 0.08 stars $/ \operatorname{arcsec}^{2}$ (considering all the detected stars). For the applications presented in this paper the effects of incompleteness are not an issue since we always compare subsets of stars that are homogeneous in magnitude and color (see Sects. 3.3 and 3.4 below). No attempt is made to determine the true Luminosity Functions of the observed sequences. The TRGB level, the main target of the present study, occurs more than 3 mag above the limiting magnitude, a range in which the considered sample is likely $\sim 100 \%$ complete. Since the photometric uncertainties of individual stars (in each passband) are empirically estimated as the standard deviations of 3 to 8 independent measures of the magnitudes there is no need for artificial star experiments to characterize photometric errors.

\subsection{Photometric calibration}

The absolute calibration has been obtained from several repeated observations of Landolt (1992) standard fields, including all the stars listed in the extended catalog of calibrators provided by Stetson (2000). The coefficients of atmospheric extinction $\left(C_{\text {ext }}\right)$ were directly obtained by repeated observations of the same standard field at different air mass. The final calibrating equations and $C_{\text {ext }}$ are shown in the upper panels of Fig. 2. 

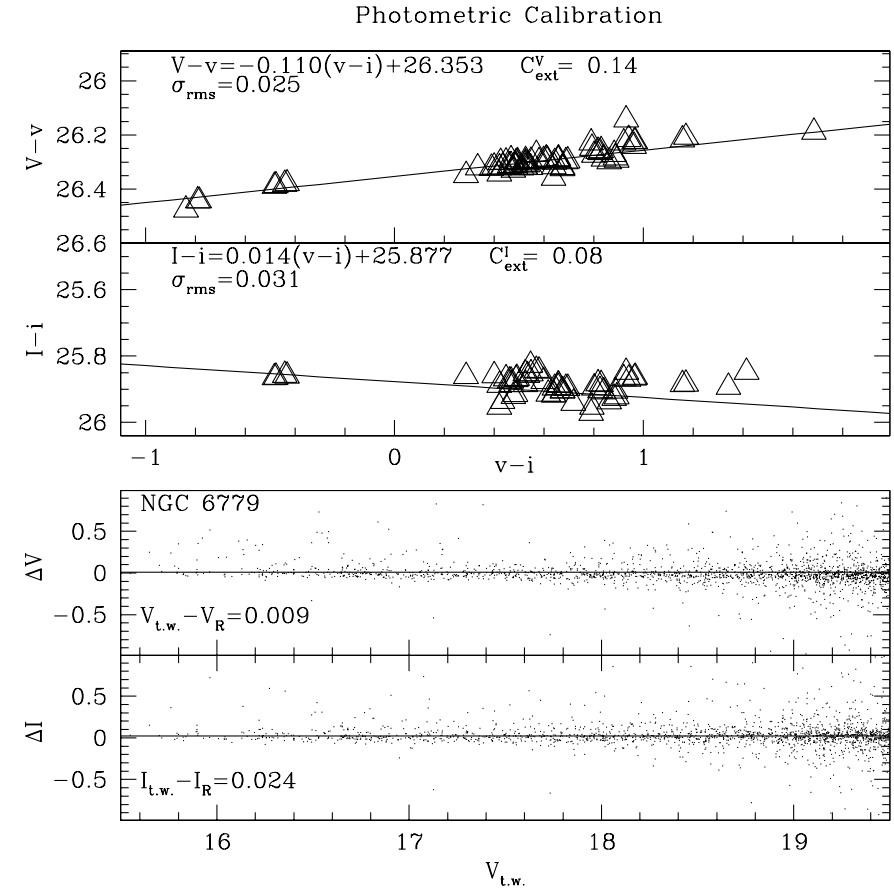

Fig. 2. Upper panels: difference between tabulated magnitudes $(V, I)$ and instrumental magnitudes $(v, i)$ vs. instrumental color index $(v-i)$ for the observed Landolt (1992) standard stars. The calibrating relations are plotted (solid lines) and the corresponding equations are reported along with the rms of the linear fits and the value of extintions coefficients. Lower panels: comparison of the photometry of NGC 6779, obtained from data acquired during the same run of the M 33 data and calibrated with the relations above, and the independent photometry by Rosenberg et al. (2000).

To check the accuracy (and reproducibility) of the photometric calibration we reduced one $V$ and one $I 30 \mathrm{~s}$ frames centered on the globular cluster NGC 6779, acquired during the second night of the run. The catalogue was calibrated with the relations shown in Fig. 2 and the final photometry was compared with the photometry of the cluster provided by Rosenberg et al. (2000). The results of the comparison (shown in the lower panels of Fig. 2) demonstrate that our photometry is in excellent agreement with that of Rosenberg et al. (2000), an the characteristic uncertainty of our absolute calibration is of the order of $\leq \pm 0.02 \mathrm{mag}$.

\section{Results}

\subsection{Color-magnitude diagram}

The Color Magnitude Diagrams (CMD) of the observed field are presented in Fig. 3. The CMD of F1 is dominated by a wide RGB sequence, running from $I \sim 20.6$ down to the limiting magnitude of the photometry. The exact location of the TRGB (as derived in Sect. 3.2, below) is reported in the CMDs as a horizontal line to the red of the observed RGB. The sources brighter and redder than the RGB Tip are likely bright Asymptotic Giant Branch (AGB) stars, probably associated with an intermediate-age (and/or metal-rich) population (see Davidge 2003, for an extensive discussion). The presence of a conspicuous plume of intermediate-young Main

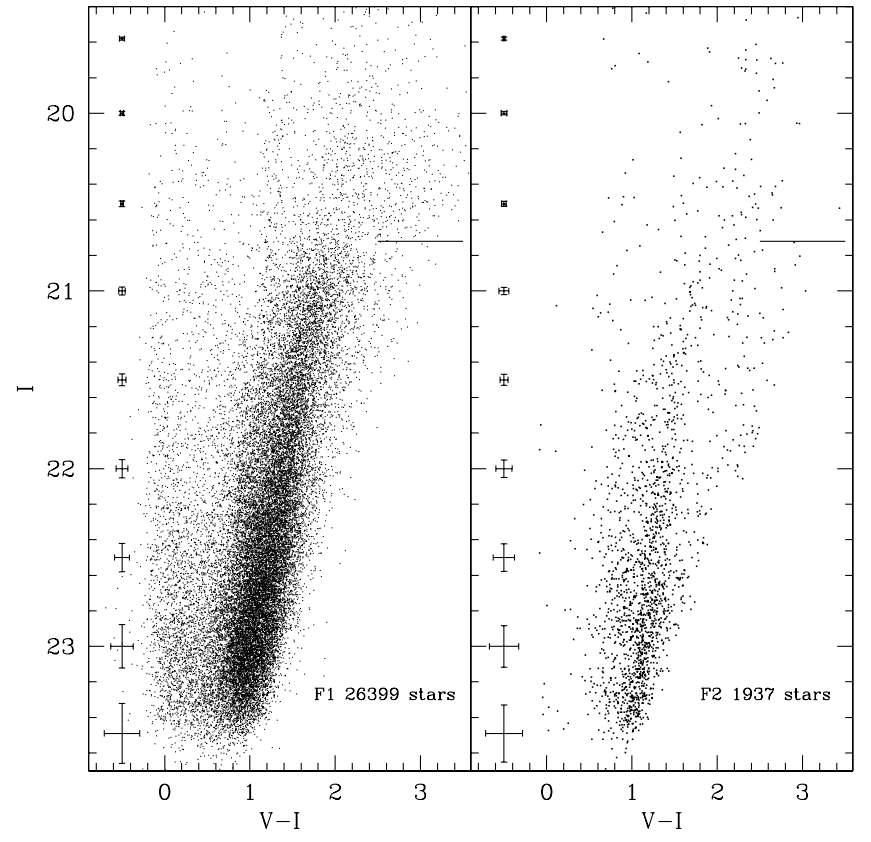

Fig. 3. Color Magnitude Diagrams of the two observed fields. The errobars show the average photometric uncertainty as a function of magnitude; the horizontal lines marks the position of the TRGB as determined in Sect. 3.2.

Sequence (MS) stars reaching $I \simeq 19.4$ is also evident on the blue side of the CMD, with a sharp blue edge at $V-I \simeq 0.0$. In the CMD of F2 only the RGB and AGB population are discernible, while the blue MS stars are completely absent. Star counts on the RGB indicate that the stellar density drops by a factor $>20$ from $F 1$ to $F 2$.

The contamination by foreground Galactic stars is negligible for the purposes of the present study. The Galactic model by Robin et al. (2003) predicts fewer than 200 Galactic stars in the observed fields within the color and magnitude ranges spanned by the CMDs of Fig. 3. By inspection of the synthetic CMD obtained with the Robin et al. (2003) model we conclude that even in the poorly populated F2 the only region of the CMD that may be significantly affected by Galactic contamination is that which hosts bright AGB stars, e.g. $V-I \gtrsim 1.8$ and $V \lesssim 20.6$ (see Sect. 3.4, below).

\subsection{TRGB distance}

The use of Tip of the Red Giant Branch (TRGB) as a standard candle is now a widely used technique to estimate the distance to galaxies of any morphological type (see Lee et al. 1993; Madore \& Freedman 1995, 1998; Walker 2003, for a detailed description of the method, recent reviews and applications). The underlying physics is well understood (Madore \& Freedman 1998; Salaris et al. 2002) and the observational procedure is operationally well defined (Madore \& Freedman 1995). The key observable is the sharp cut-off occurring at the bright end of the RGB Luminosity Function (LF) that can be easily detected with the application of an edge-detector filter (Sobel filter, Madore \& Freedman 1995; Sakai et al. 1996) or by other (generally parametric) techniques (see, for example 
Méndez et al. 2002; McConnachie et al. 2004). The necessary condition for a safe application of the technique is that the observed RGB Luminosity Function should be well populated, with more than $\sim 100$ stars within 1 mag from the TRGB (Madore \& Freedman 1995; Bellazzini et al. 2002).

The F2 sample is not sufficiently populated for a safe application of the method while the F1 sample clearly fulfils the above criterion (there are more than 2500 RGB stars within 1 mag from the TRGB), hence we limit the TRGB research to F1. As a first step, to limit the range of metallicity of the stars involved in the TRGB detection, we select RGB stars by color following the approach adopted by McConnachie et al. (2004). The adopted selection includes the main bulk of the RGB population and it is shown in the lower left panel of Fig. 4. The logarithmic LF is presented as an ordinary histogram and as a generalized histogram (e.g. the histogram convolved with a Gaussian with standard deviation equal to the photometric error at the given magnitude, see Laird et al. 1988; Bellazzini et al. 2002, for definitions and references) in the upper left and upper right panels of Fig. 4, respectively. The sharp cut-off is an obvious feature of both representations of the LF and is easily detected by the Sobel filter (Fig. 4, lower right panel). As usual, the peak of the filter response is taken as the best estimate of the TRGB location and the Half Width at Half Maximum of the same peak is taken as the associated uncertainty, $I^{\mathrm{TRGB}}=20.72 \pm 0.08$. If we consider the most recent estimates in the literature, our value is $\sim 2.2 \sigma$ larger than that found by McConnachie et al. $\left(2004, I^{\mathrm{TRGB}}=20.54 \pm\right.$ 0.01 ; but these authors provide only a formal error on their estimate), and $\sim 1-2 \sigma$ lower than the estimates by Kim et al. $\left(2002, I^{\text {TRGB }}=20.82-20.92 \pm 0.05\right.$ depending on the considered field), e.g. it is bracketed by the two quoted results. On the other hand our estimate is in excellent agreement with that obtained by $\mathrm{T} 04\left(I^{\mathrm{TRGB}}=20.75 \pm 0.02\right)$.

We adopt $E(B-V)=0.04$, according to the reddening maps by Schlegel et al. (1998) and Burstein \& Heiles (1984) and $A_{I}=1.76 E(B-V)$, according to Dean et al. (1978). We note however that most of the other available estimates of the foreground reddening cluster around $E(B-V)=0.08$ (see Van den Bergh 1991, and references therein). To account for this, we report also the results we obtain adopting $E(B-V)=$ 0.08 (see Table 2, below). Note that the effects of this different assumption are small either on the final distance modulus (e.g. $0.05 \mathrm{mag})$ or the average metallicity $(\leq 0.15 \mathrm{dex}$; see Table 2$)$. According to the detailed dust maps of M 33 by Hippelein et al. (2003) the effect of the intrinsic extinction should be negligible in the fields considered here.

In Bellazzini et al. (2001) we have provided a robust zeropoint to the calibrating relation providing the absolute $I$ magnitude of the tip $\left(M_{I}^{\mathrm{TRGB}}\right)$ as a function of metallicity $([\mathrm{Fe} / \mathrm{H}]$, in the Zinn \& West (1984) scale, hereafter ZW), based on the geometric distance to the cluster $\omega$ Centauri obtained by Thompson et al. (2001) using the double-lined detached eclipsing binary OGLE-17. This calibration is fully independent of the usual (Cepheid based and/or RR Lyrae based) distance scales. In Bellazzini et al. (2004) we have extended the calibration to Near Infrared passbands and we refined our $I$ calibration providing also the relation for $M_{I}^{\mathrm{TRGB}}$ as a function of the
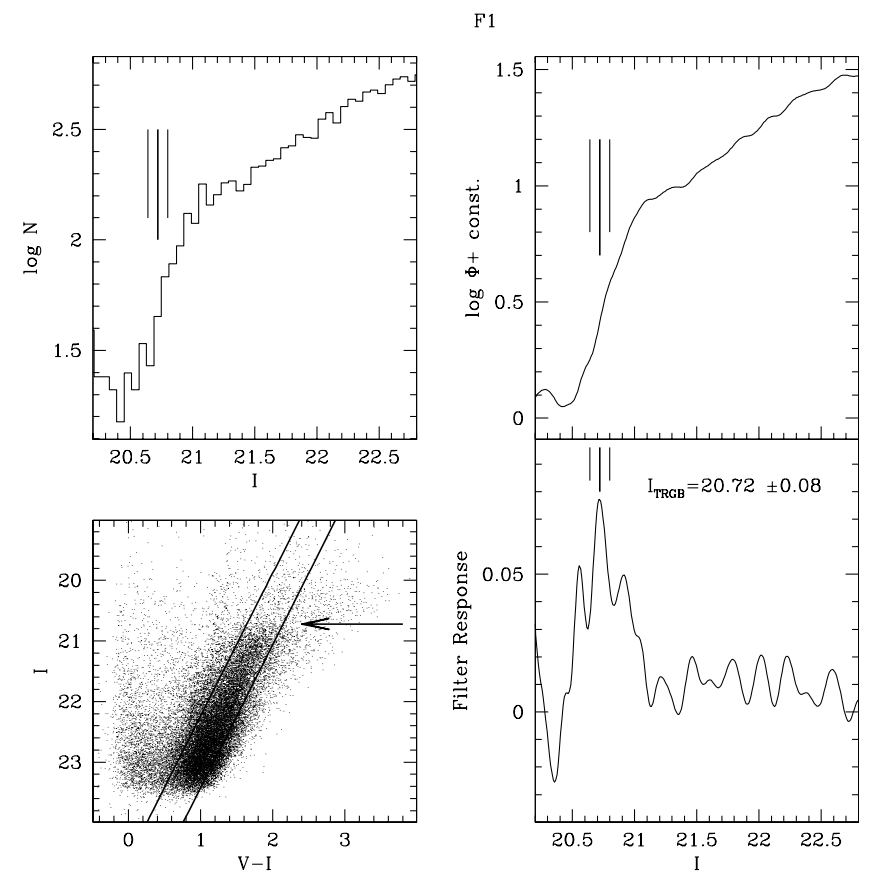

Fig. 4. Detection of the TRGB. The CMD in the lower left panel shows the adopted selection, e.g. the stars enclosed by the two diagonal lines. The arrow marks the position of the TRGB. The upper panels display the logarithmic LF of the upper RGB as an ordinary histogram (left) and as a generalized histogram (right). The thick lines marks the position of the TRGB, the thin lines enclose the associate uncertainty range. Lower panel: response of the Sobel's filter to the observed LF.

global metallicity $([M / \mathrm{H}]$, see Salaris et al. (1993) and Ferraro et al. (1999) for definitions and discussion) that we adopt in the present analysis.

Since the distance modulus derived from $I^{\mathrm{TRGB}}$ is weakly dependent on metallicity, and our metallicity estimates (obtained by comparison with template RGB ridge lines, see below) depend on the assumed distance modulus, we adopted an iterative method to find simultaneously the two quantities searched for. First we derived a preliminary distance modulus adopting $M_{I}^{\mathrm{TRGB}}=-4.04$, then we derived a median metallicity of the considered population as described in Sect. 3.3 below, and we obtained a refined estimate of the modulus using the obtained median metallicity $([M / \mathrm{H}]=-0.75$, see below) as an input for the calibrating relation by Bellazzini et al. (2004):

$$
M_{I}^{\mathrm{TRGB}}=0.258[M / \mathrm{H}]^{2}+0.676[M / \mathrm{H}]-3.629( \pm 0.12) .
$$

The process converged to stable values of the distance modulus and of the median metallicity in 2-3 iterations, independently of the assumed reddening and/or the considered metallicity scale. Our final estimate (for $E(B-V)=0.04$ and $\left.[M / \mathrm{H}]_{\text {med }}=-0.75\right)$ is $(m-M)_{0}=24.64 \pm 0.15$, where all the sources of uncertainty have been taken into account. The corresponding distance estimate is $D=847 \pm 60 \mathrm{kpc}$.

\subsection{Metallicity}

We derive the Metallicity Distributions (MD) of the studied fields from the Color and Magnitude distribution of RGB stars 

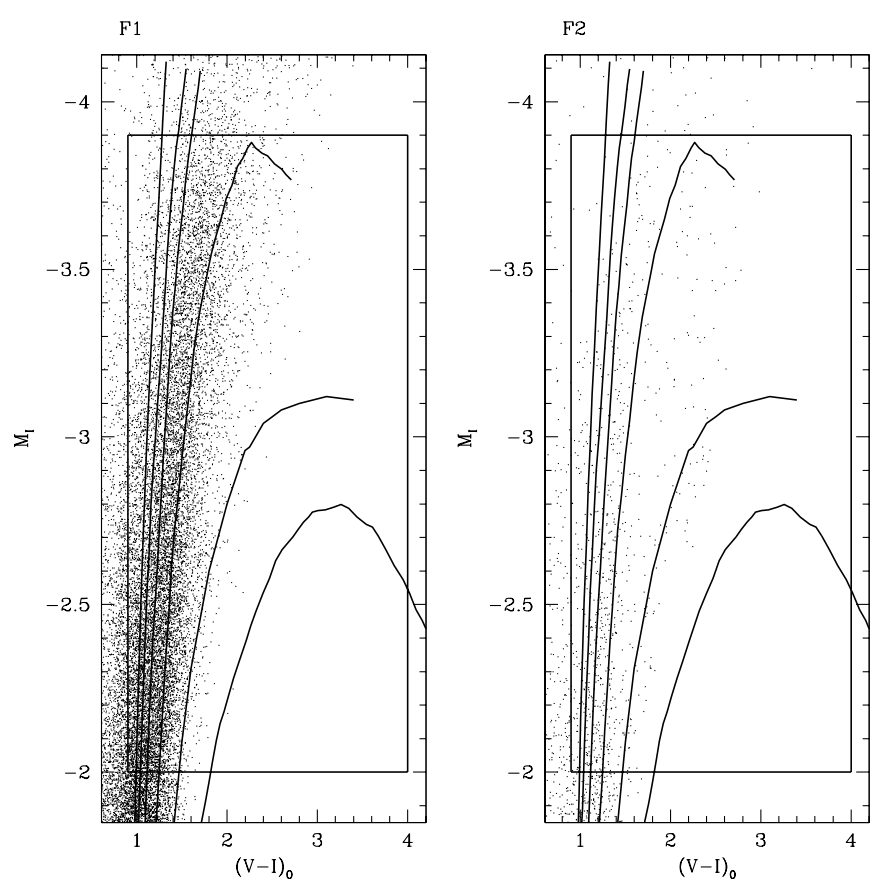

Fig. 5. The CMDs of the RGB stars in F1 (left panel) and F2 (right panel) are compared to the grid of RGB ridge lines of template globular clusters we adopted to derive the metallicity distributions. From blue to red, the template clusters are: M 92, M 13, M 5, 47 Tuc, NGC 6553 and NGC 6528. The thick box encloses the RGB stars actually selected for the derivation of the metallicity distribution (see Bellazzini et al. 2003, for details).

(transformed to the absolute $(V-I)_{0}$ vs. $M_{I}$ plane adopting the reddening and distance modulus described above) by interpolation on a grid of RGB ridgelines of template globular clusters, adopting the same scheme as Bellazzini et al. (2003). Essentially the same approach is adopted also by T04. The MDs are obtained in different metallicity scales, e.g. the ZW scale, the scale by Carretta \& Gratton (1997, hereafter CG) and the global metallicity scale described in Ferraro et al. (1999), to make the comparison with other studies easier. While the individual photometric metallicities provided by the adopted procedure may be quite uncertain, the overall metallicity distribution and its average properties are sufficiently well characterized to provide interesting insights into the stellar population under analysis and it has been widely used, in recent years, in the study of resolved galaxies (see Bellazzini et al. 2003, for details, discussion and references). The color distribution of RGB stars should depend - to a lesser extent also on the age distribution of the underlying population. T04 studied this problem in the case of M 33 by mean of synthetic CMDs drawn from theoretical evolutionary tracks and concluded that "...the ages of the RGB stars are not likely to significantly affect the derived MDs...". They estimate that the maximum expected shift of the peak of the MDs is of the order of $0.1-0.2$ dex. Finally, the young MS population observed in F1 and not in F2 - cannot affect the comparison between the MDs of the two fields since their evolved counterparts should be negligible in number and do not fall in the selection box we adopt to obtain MDs (see Figs. 5 and 7, below). When dealing with

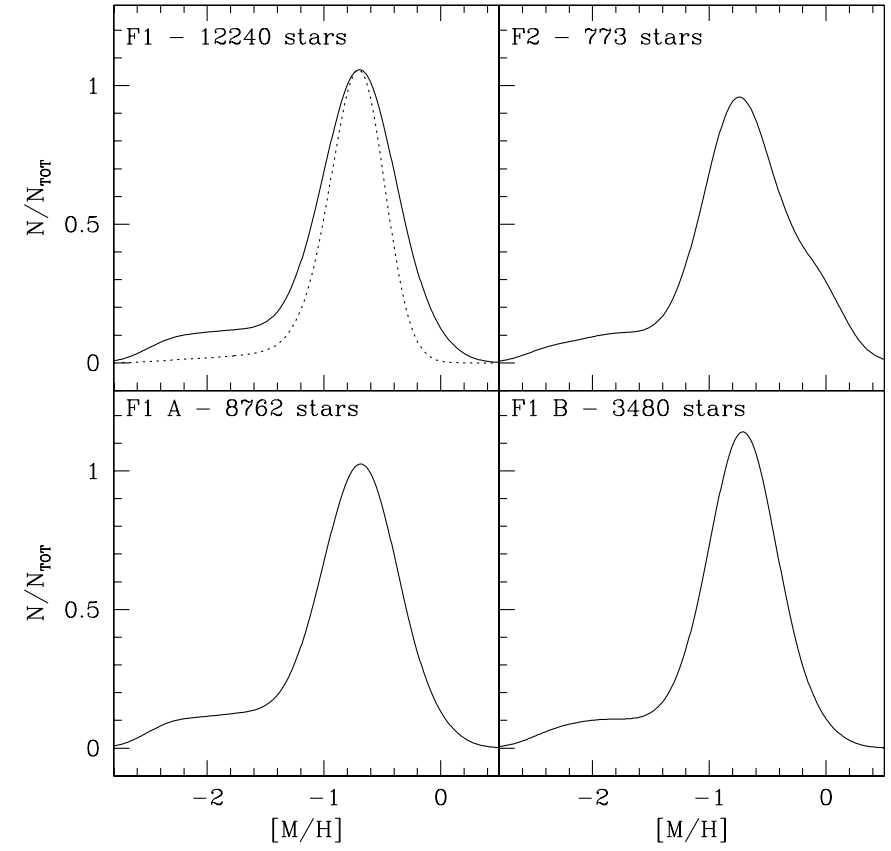

Fig. 6. Metallicity Distributions (continuous lines) of the F1 field as a whole (upper left panel), of F2 (upper right panel), of the inner part of F1 $\left(10^{\prime} \lesssim R \lesssim 15^{\prime} ; \mathrm{F} 1 \mathrm{~A}\right.$, lower left panel) and of the outer part of the same field $\left(15^{\prime} \lesssim R \lesssim 20^{\prime} ; \mathrm{F} 1 \mathrm{~B}\right.$, lower right panel). The dotted line in the upper left panel shows the instrumental response of the method, the MD that would be obtained if the width of the RGB was entirely due to the photometric errors.

photometric metallicities it should be kept in mind that the underlying age distribution may affect the derived MDs.

In Fig. 5 a direct comparison between the observed RGBs of F1 and F2 and the adopted template ridge lines is presented. It is immediately clear that the large majority of M 33 RGB stars (in both fields) are enclosed within the ridge lines of $\mathrm{M} 5\left([\mathrm{Fe} / \mathrm{H}]_{C G}=-1.11 ;[M / \mathrm{H}]=-0.90\right)$ and of 47 Tuc $\left([\mathrm{Fe} / \mathrm{H}]_{C G}=-0.70 ;[M / \mathrm{H}]=-0.60\right)$. In the CMD of $\mathrm{F} 2$ an anomalous clustering of stars can be noted around the ridge line of NGC $6553\left([\mathrm{Fe} / \mathrm{H}]_{C G}=-0.16 ;[M / \mathrm{H}]=-0.06\right)$ that has no counterpart in the CMD of F1. However, as can be appreciated from Fig. 3, such red stars are just above the limiting magnitude at their color. For this reason we will not discuss in detail this feature in the following. Deeper photometry is needed to firmly assess the possible presence of an excess of very red RGB stars in this region.

In Fig. 6 we present the MDs (in the form of generalized histograms) as a function of the global metallicity for F1 (upper left panel) and F2 (upper right panel). To study in finer detail the radial behavior of he MD we split F1 in two subregions (A and B) of similar area. In particular F1 A contains all the F1 stars less distant than $15^{\prime}$ from the center of M 33, while the F1 stars with $r \geq 15^{\prime}$ are assigned to F1 B. The MDs of F1 A and F1 B are plotted in the lower left and lower right panels of Fig. 6, respectively. Note that if we exclude from the interpolation the stars with $M_{I} \geq-2.5$, i.e. in the range where the sensitivity of color to metallicity is lower, the obtained MDs are unchanged. This experiment also demonstrates that the derived MD are not sensitive to the effect of 
Table 2. Distance modulus, median metallicity and standard deviations in different metallicity scales, and with different assumptions on the reddening. Note that the median and the mean metallicity are nearly coincident in all the considered cases.

\begin{tabular}{l|rr|rr}
\hline \hline & \multicolumn{2}{|c|}{$\mathrm{F} 1$} & $\mathrm{~F} 2$ & \multicolumn{2}{c}{$\mathrm{F} 1$} & $\mathrm{~F} 2$ \\
\hline$E(B-V)$ & \multicolumn{2}{|c|}{0.04} & \multicolumn{2}{c}{0.08} \\
\hline$[\mathrm{Fe} / \mathrm{H}]_{Z W}$ & -1.03 & -1.03 & -1.17 & -1.18 \\
$\sigma_{Z W}$ & 0.40 & 0.40 & 0.40 & 0.44 \\
{$[\mathrm{Fe} / \mathrm{H}]_{C G}$} & -0.89 & -0.89 & -0.97 & -0.98 \\
$\sigma_{C G}$ & 0.27 & 0.28 & 0.28 & 0.32 \\
{$[M / \mathrm{H}]$} & -0.75 & -0.74 & -0.81 & -0.81 \\
$\sigma_{[M / \mathrm{H}]}$ & 0.23 & 0.23 & 0.23 & 0.27 \\
\hline$(m-M)_{0}$ & \multicolumn{2}{|c|}{$24.64 \pm 0.15$} & \multicolumn{2}{|c}{$24.59 \pm 0.15$} \\
\hline
\end{tabular}

incompleteness, as expected. The average properties of all the considered MDs are summarized in Table 2, for two different assumptions of $E(B-V)$. There are a number of considerations emerging from the inspection of Fig. 6 and Table 2:

1. The MD of all the considered fields shows a strong peak at $[M / \mathrm{H}] \simeq-0.7$. This justifies our assumption of the median metallicity as the characteristic value of the dominant population in our determination of the TRGB distance. A sparsely populated tail of metal-poor stars extending to $[M / \mathrm{H}]<-2.0$ is also present in all the presented MDs. This general similarity over large areas of the galaxy (F1 covers a range of galactocentric distances from $\sim 2.4 \mathrm{kpc}$ to $\sim 5 \mathrm{kpc}, \mathrm{F} 2$ from $\sim 5.5 \mathrm{kpc}$ to $\sim 8.2 \mathrm{kpc}$ ) is in agreement with the results by Kim et al. (2002) and is reminiscent of what is observed in M 31 (Bellazzini et al. 2003). Our MDs are very similar to those obtained by $\mathrm{T} 04$.

2. The bell-shaped curve plotted as a dotted line in the upper left panel of Fig. 6 displays the response of the adopted interpolation scheme to a Simple Stellar Population (SSP, i.e. a population of stars having the same age and chemical composition, Renzini \& Fusi Pecci 1988) observed under the same conditions as our real data. It has been obtained adopting, as input for the interpolation, a synthetic RGB population whose color width is entirely due to the photometric errors. The I magnitude of the "synthetic" stars is extracted from the observed RGB luminosity function, the $V-I$ color is obtained from the average ridge line of the observed RGB plus a photometric error drawn at random from a Gaussian distribution having $\sigma$ equal to the average observed photometric uncertainty at the considered magnitude (as done in Bellazzini et al. 2002).

To obtain the true width of the underlying MD the described "instrumental response" should be deconvolved from the observed MD (continuous line). The main peak of the observed MD is well fitted by a Gaussian distribution with $\sigma=0.34$ dex, while the instrumental response curve is well approximated by a Gaussian distribution with $\sigma=0.23$ dex. It may be concluded that the true intrinsic dispersion of the main peak of the MD is $\sigma \simeq 0.25 \mathrm{dex}$.

3 . By comparison with ridge lines of template globular clusters, Mould \& Kristian (1986) estimated
$\left\langle[\mathrm{Fe} / \mathrm{H}]_{Z W}\right\rangle \simeq-2.2 \pm 0.8$ for a field at a galactocentric distance similar to F2. On the other hand, we find, for both $\mathrm{F} 1$ and $\mathrm{F} 2,\left\langle[\mathrm{Fe} / \mathrm{H}]_{Z W}\right\rangle \simeq-1.03 \pm 0.40$, in excellent agreement with the results by Davidge (2003), Cuillandre et al. (1999) and T04. The difference is partly justified by the different assumptions about distance $\left(\left(m-M_{0}\right)=24.8\right.$ instead of our $\left.\left(m-M_{0}\right)=24.64\right)$. However, even adopting their distance modulus we find $\left\langle[\mathrm{Fe} / \mathrm{H}]_{Z W}\right\rangle \simeq-1.22 \pm 0.40$, much more metal-rich than what was found by Mould \& Kristian (1986). We tentatively ascribe this difference to a possible problem in the absolute calibration of Mould \& Kristian's photometry. This hypothesis is confirmed by the results of T04.

4. Assuming the same distance modulus as Mould \& Kristian (1986), Kim et al. (2002) obtain $-0.61 \leq\left\langle[\mathrm{Fe} / \mathrm{H}]_{Z W}\right\rangle \leq$ -0.86 for 10 fields covering (approximately) the same radial range than our F1. The agreement with our results is much better than with Mould \& Kristian's one, still the difference is not negligible and it is not justified by the different distance modulus assumed (a larger distance modulus should imply a brighter and hence more metal-poor RGB). However Kim et al. (2002) derived their mean metallicity from the mean $(V-I)_{0}$ color at $M_{I}=-3.5$. We consider our median/mean metallicities, derived from all the RGB stars brighter than $M_{I}=-2.0$ and based on an accurately checked photometric calibration, as more robust and safer than those by Kim et al. (2002).

5. Kim et al. (2002) found a weak radial gradient in the mean metallicity $(0.2 \mathrm{dex})$ in the range $1 \leq R \leq 5 \mathrm{kpc}$ (see also T04). We find no sign of variation of the mean metallicity in the range $2.4 \leq R \leq 8.2 \mathrm{kpc}$, but such feeble differences may have gone undetected at the level of accuracy of our relative photometry (e.g. may be hidden in the "instrumental width" of the observed sequences).

6. The weak shoulder at $[M / \mathrm{H}] \sim-0.2$ in the MD of $\mathrm{F} 2$ is due to the handful of red stars around the ridge line of NGC 6553 discussed above, hence, at the present stage, cannot be trusted as a real feature of the MD (but it deserves further investigation).

\subsection{Stellar populations and radial distributions}

The stellar content of the outer region of M 33 is poorly explored. It is interesting to investigate if the different kind of stars identified in our CMDs share the same spatial distribution. To check this point we defined three selection boxes that are depicted in the F1 CMD shown in Fig. 7. Note that the boxes are defined in a range of magnitude in which incompleteness effects should be weak or negligible $\left(M_{I}<-3.0\right.$, e.g. more than 2 mag above the limiting magnitude) and cover similar magnitude ranges.

As a guideline, we have superimposed three isochrones of solar metallicity and age $=20,40,100 \mathrm{Myr}$ (continuous lines) and three isochrones with $[M / \mathrm{H}]=-0.7$ and age $=2,6,12 \mathrm{Gyr}$ (empty symbols) from the set by Girardi et al. (2000). The bluest box samples the upper MS, e.g. young stars with age $\leq$ 100 Myr. The large box above the TRGB samples the bright 


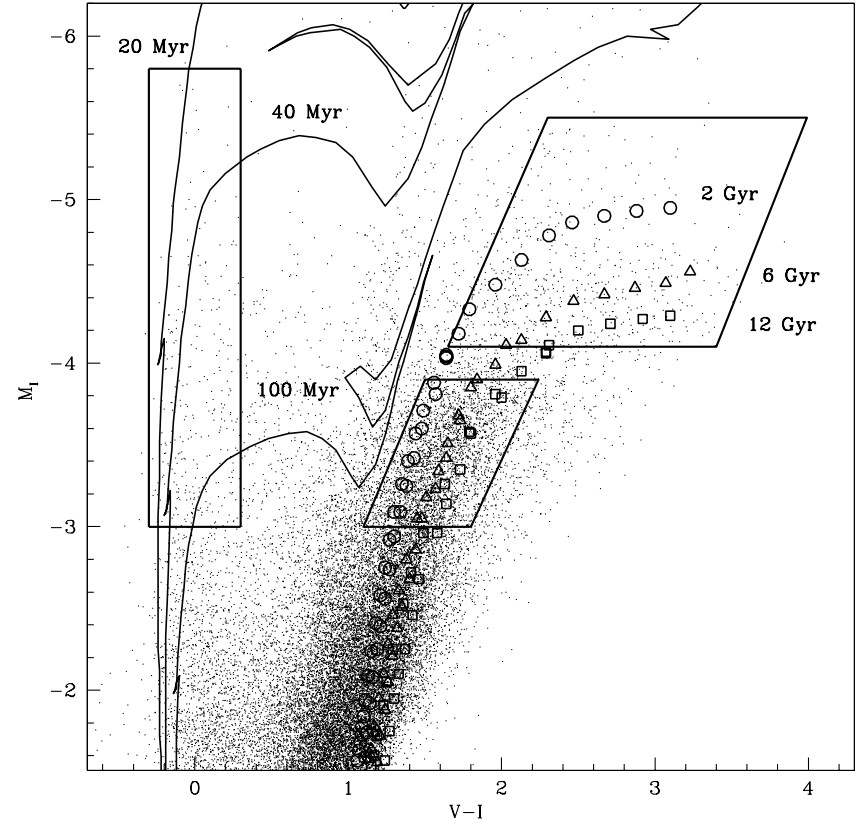

Fig. 7. Description of the adopted selection boxes. The box around $V-I=0.0$ selects a sample of young MS stars, the large box above the TRGB selects AGB stars, the small box below the TRGB selects RGB stars. The continuous lines are isochrones of solar metallicity, the open symbols are isochrones at $[M / \mathrm{H}]=-0.7$, from the set by Girardi et al. (2000). The ages are reported in the plot. The small dots are the observed stars of F1.

AGB stars, the smaller box samples the brightest RGB stars. Both kinds of tracers are associated with intermediate to old age stars, but are not necessarily linked thogether. In particular, bright RGB stars trace populations older than 1-2 Gyr (see Salaris et al. 2002, and references therein). In the following, $R$ must be intended as the projected angular distance from the center of the galaxy.

In the upper panels of Fig. 8 the adopted selection boxes are superposed on the CMDs of F1 (left panel) and F2 (right panel). The lower panels of Fig. 8 show the cumulative radial distributions of the stars falling in the boxes in the two different fields. In the radial range covered by $\mathrm{F} 1$ the distributions of RGB and AGB stars are indistinguishable. On the other hand, MS stars appear much more centrally concentrated since their distribution seems to end at $R \sim 17^{\prime}-18^{\prime}$, e.g. around 2 disc scalelengths (Van den Bergh 1991). According to a KolmogorovSmirnov test, the probability that the MS and RGB samples are drawn from the same parent population is $P \leq 0.04 \%$. This suggests that the RGB and AGB stars are not associated with the young disc component traced by MS stars. At least a (significant) fraction of them should belong to a more extended galactic component.

The radial distribution of MS stars is not reported in the lower right panel of Fig. 8 since, in agreement with the above conclusion, F2 is virtually devoid of stars populating the MS box. It is surprising to note that in this field, AGB stars appear to follow a significantly different distribution with respect to RGB stars. The distribution of AGB stars is less centrally concentrated and it is quite similar to uniform distribution on the sky. The latter fact would be naturally explained if the
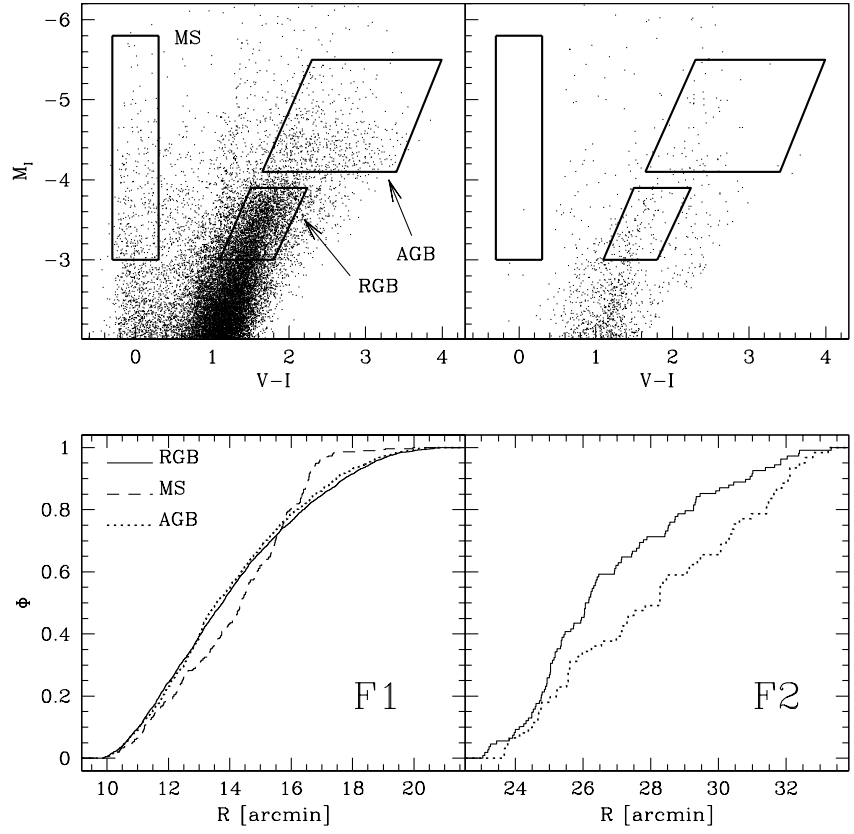

Fig. 8. Upper panels: the selection boxes described in Fig. 7 are superposed on the CMDs of F1 (left) and F2 (right). The corresponding cumulative radial distributions are displayed in the lower panels.

AGB sample of F2 would be dominated by foreground contamination (see also Davidge 2003). According to the predictions of the Galactic model by Robin et al. (2003) this seems to be the actual case. The model predicts that the number of Galactic stars falling in the AGB box is 48 , less than compared to the 61 actually observed. Hence $\simeq 80 \%$ of the putative AGB stars in F2 are likely foreground stars. The impact is much smaller on the F1 stars where 584 stars are observed in the AGB box, hence the fraction of foreground contaminants is $<10 \%$. On the other hand the expected number of foreground stars falling in the RGB box is $\sim 4$, e.g. negligible in both fields.

Therefore, the difference of radial distribution shown in the lower right panel of Fig. 8 is completely spurious. On the other hand, if we consider star counts and take into account the corrections for foreground contamination, it turns out that while the number of RGB stars drops by a factor $\simeq 27$ going from $F 1$ to F2, the number of AGB stars decreases by a larger factor, $\simeq 42$. This suggests that RGB stars may follow a more extended distribution with respect to AGB stars at large radii.

All the above considerations seem to indicate that populations of different characteristic ages follow different distributions on the sampled scales, the older stars having more extended distributions. This is suggestive of the presence of a weak "classical" old halo component in M 33. The only previous indication in this sense (from field stars) is provided by the discovery of a few candidate RR Lyrae variables by Pritchet (1988), while all other hints of the existence of an old spheroidal stellar component come from the study of globular clusters (Schommer et al. 1991; Sarajedini et al. 2000). Note, however, that an extended and old disc component is also compatible with our observations (see T04).

In the upper panel of Fig. 9 we report the radial profiles of RGB and MS stars over the range(s) sampled by the present 

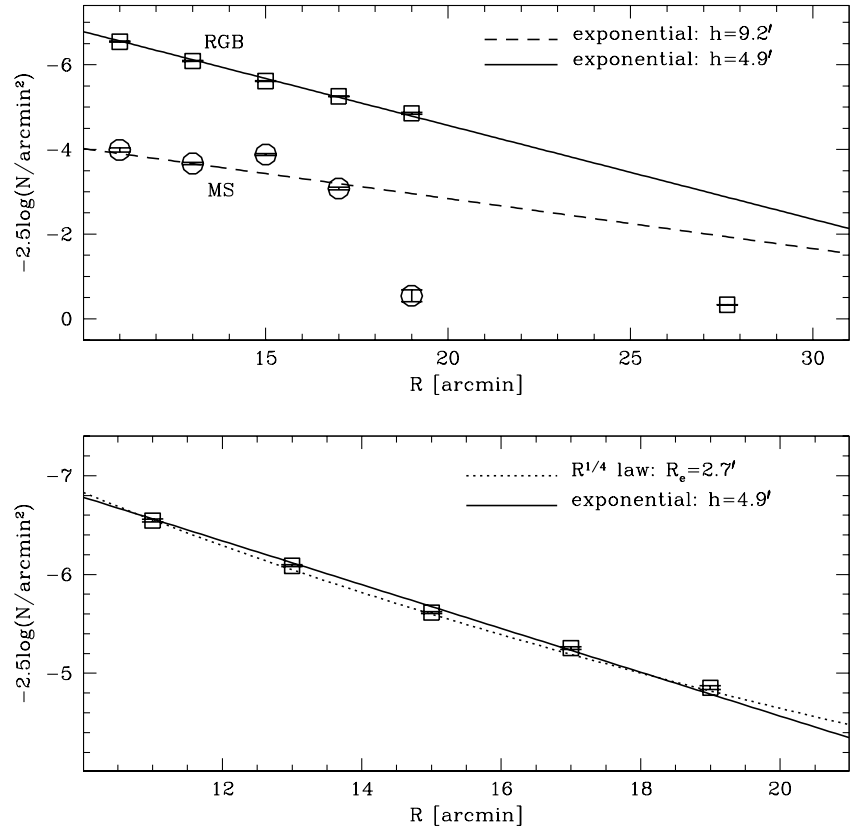

Fig. 9. Upper panel: stellar density profiles for MS (open circles) and RGB stars (open squares) compared with two different exponential laws. All the estimates with $R \leq 20^{\prime}$ are computed over sections of concentric annuli $2^{\prime}$ wide. The RGB points at $R=27.6^{\prime}$ is the density estimate obtained from F2 as a whole. Lower panel: expansion of the RGB density profile. The continuous line is the same exponential law displayed in the upper panel, the dotted line is a $R^{\frac{1}{4}}$ law with $R_{\mathrm{e}}=$ 9.2' . The associated uncertainty is the Poisson noise of the star counts propagated to the adopted unities.

study. The MS profile is reasonably reproduced by an exponential law with scale-length $h=9.2^{\prime}$ up to $R \simeq 17^{\prime}$, in agreement with the results by Kent (1987). However, at $R=19^{\prime}$ the sharp drop of the density already observed and discussed in Fig. 8 is clearly evident. On the other hand the RGB profile is well fitted by an exponential law with $h=4.9^{\prime}$ over the whole radial range sampled by $\mathrm{F} 1$. The density of RGB stars falls significantly below the adopted exponential profile in F2, suggesting a break in the observed profile in the range $20^{\prime} \lesssim R \lesssim 25^{\prime}$. In our view, the most interesting result of this comparison is that, even ignoring the observed density cut-offs, the RGB and MS distributions do have significantly different profiles, again suggesting a different origin.

The lower panel of Fig. 9 shows that in the radial range covered by $\mathrm{F} 1\left(10^{\prime} \leq R \leq 20^{\prime}\right)$ the observed density profile of RGB stars is equally well fitted by the exponential law described above and by a $R^{\frac{1}{4}}$ law having effective radius $R_{\mathrm{e}}=2.7^{\prime}$ (in fact, the $R^{\frac{1}{4}}$ law provides a marginally better fit with respect to the exponential). It is interesting to note that the same $R^{\frac{1}{4}}$ law was found to provide a good fit also to the central bulge of M 33 (Boulesteix et al. 1980; Bothun 1992), suggesting a possible connection between the bulge and the putative halo component (but see Stephens \& Frogel 2002, for a detailed decomposition of the inner profile). While suggestive, the above result is limited to the considered radial range $\left(10^{\prime} \leq R \leq 20^{\prime}\right)$ where the contribution of disc stars to the RGB population may be low. To correctly disentangle the contribution of the disc from that

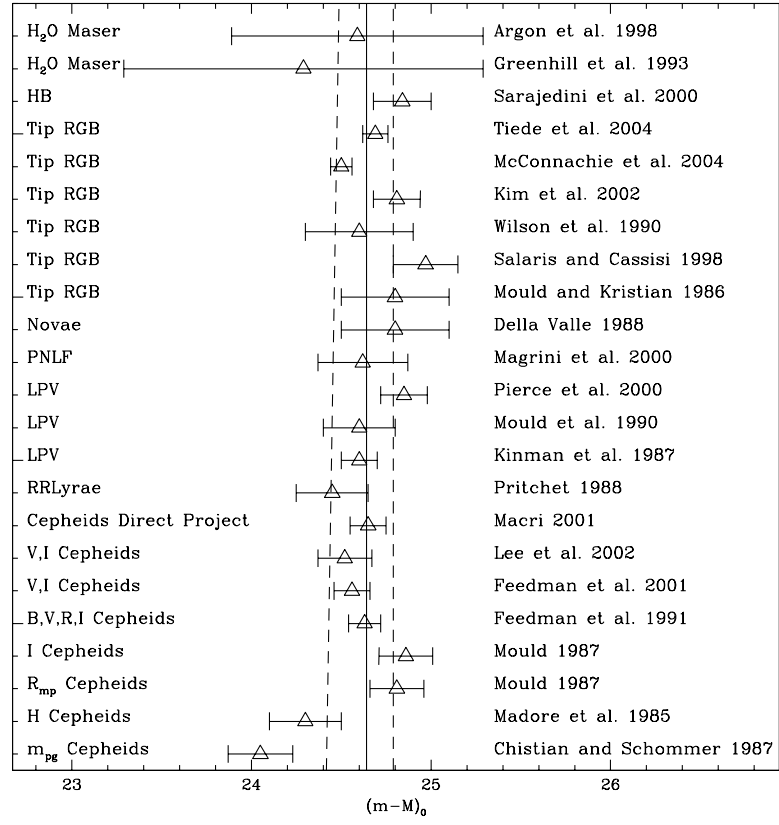

Fig. 10. Previous estimates of the distance modulus from the literature (open triangles) are compared with the estimate obtained in the present study (continuous vertical line). The dashed line encloses the uncertainty range of our estimate. The method adopted in the different estimate is indicated on the left part of the plot, the sources on the right part. $\mathrm{HB}=$ Horizontal Branch; PNLF = Planetary Nebulae Luminosity Function; LPV = Long Period Variables.

of the more extended component identified here, a complete sampling of the density profile from the center to the outskirts of the galaxy is needed, e.g. covering also the regions in which the surface brightness should be dominated by the exponential disc. This kind of analysis is clearly beyond the reach of the present study.

\section{Conclusions}

We have obtained a robust detection of the $I$ magnitude of the TRGB in a field located at $\sim 15^{\prime}$ from the center of $M$ 33, near the galaxy minor axis. Adopting the median metallicity we derived from the same data and the calibration of $M_{I}^{\mathrm{TRGB}}$ as a function of the global metallicity $([M / \mathrm{H}])$ provided by Bellazzini et al. (2004), we have obtained a new estimate of the distance modulus of $\mathrm{M} \mathrm{33,}(m-M)_{0}=24.64 \pm 0.15$. All the sources of uncertainty have been taken into account in the reported error bar.

In Fig. 10, our distance modulus is compared with previous estimates available in the literature. The large majority of the reported values are compatible, within the formal $1-\sigma$ errors, with our estimate. The only exceptions are provided by the oldest analysis of Cepheid variables, based on photographic plates (Madore et al. 1985; Christian \& Schommer 1987). If we exclude these two estimates as well as those by Argon et al. (1998) and Greenhill et al. (1993), that are affected by very large uncertainties, we obtain, from 19 independent estimates including ours, an average distance modulus ( $m-$ $M)_{0}=24.69 \pm 0.15$ (average \pm standard deviation) in excellent 
agreement with our result. It is interesting to note that the standard error on the above average is just $\epsilon=\sigma / \sqrt{19}=0.03$ mag.

The photometric metallicity distributions described in Sect. 3.3 indicate that (a) the observed RGB population in the outskirts of M 33 has a typical metallicity $\left([\mathrm{Fe} / \mathrm{H}]_{Z W} \simeq-1.0\right)$ that is intermediate between that of the halo of the Milky Way $\left([\mathrm{Fe} / \mathrm{H}]_{Z W} \simeq-1.5\right)$ and that of M $31\left([\mathrm{Fe} / \mathrm{H}]_{Z W} \simeq-0.6\right)$ (see Bellazzini et al. 2003, for discussion and references); (b) the MDs are quite similar everywhere, within the sampled regions, similar to the case of M 31 (Bellazzini et al. 2003).

In the radial range $10^{\prime} \leq R \leq 20^{\prime}$, the AGB and RGB stars have a similar radial distribution, much more extended than that of young MS stars which appear to decrease abruptly around $R=18^{\prime}$. The distribution of RGB stars is equally well fitted by an exponential law (but not the same that fits the distribution of MS stars) or by the same $R^{1 / 4}$ law that fits the central bulge (Boulesteix et al. 1980). The density of RGB stars is observed to fall far below that predicted by both the adopted bestfit models at $R \sim 27^{\prime}$, but the actual break may occur anywhere between $R=20^{\prime}$ and $R=27^{\prime}$ (e.g. between 4 and $\sim 5$ exponential scalelenghts, in good agreement with the complete profile obtained by Ferguson et al. (2004) from the huge database presented in McConnachie et al. (2004); A. Ferguson, private communication). The above results may suggest that a weak oldhalo component is indeed present also in M 33, but probably the point could be established only with an analysis of the stellar kinematics in the considered radial ranges. The possibility to interpret the observations of the stellar content at large distance from the center of the galaxy in terms of an unexpectedly metal rich halo or of a very extended old disc is (again) reminescent of the case of M 31 (see Ferguson \& Johnson 2001; Bellazzini et al. 2003, for discussions and references).

Acknowledgements. This research is partially supported by the Italian Ministero dell'Universitá e della Ricerca Scientifica (MURST) through the COFIN grant p. 2002028935-001, assigned to the project Distance and stellar populations in the galaxies of the Local Group. This work was supported by a fellowship (S.G.) from the Consorzio Nazionale Astronomia ed Astrofisica-CNAA and contributions from MIUR-COFIN. Part of the data analysis has been performed using software developed by P. Montegriffo at the INAF - Osservatorio Astronomico di Bologna. This research has made use of NASA's Astrophysics Data System Abstract Service. The kind assistance of the TNG staff during the observing run is also acknowledged.

\section{References}

Argon, A. L., Greenhill, L. J., Moran, J. M., Reid, M. J., \& Menten, K. M. 1998, IAU Coll., 164, Radio Emission from Galactic and Extragalactic Compact Sources, ed. J. A. Zensus, G. B. Taylor, \& J. M. Wrobel, ASP Conf. Ser., 144, 235

Bellazzini, M., Ferraro, F. R., \& Pancino, E. 2001, ApJ, 556, 635

Bellazzini, M., Ferraro, F. R., Origlia, L., et al. 2002, AJ, 124, 3222

Bellazzini, M., Cacciari, C., Federici, L., Fusi Pecci, F., \& Rich, M. 2003, A\&A, 405, 867

Bellazzini, M., Ferraro, F. R., Sollima, A., Pancino, E., \& Origlia, L. 2004, A\&A, in press [arXiv: astro-ph/0404572]

Bothun, G. D. 1992, AJ, 103, 104

Boulesteix, J., Colin, J., Athanassoula, E., \& Monnet, G. 1980, in Photometry, Kinematics and Dynamics of Galaxies, 271
Burstein, D., \& Heiles, C. 1984, ApJS, 54, 33

Carretta, E., \& Gratton, R. 1997, A\&AS, 121, 95

Christian, C. A., \& Schommer, R. A. 1987, AJ, 93, 557

Cuillandre, J.-C., Lequeux, J., \& Loinard, L. 1999, in The stellar content of the Local Group, ed. P. Whitelock, \& R. Cannon (S. Francisco: ASP), IAU Symp., 192, 185

Davidge, T. J. 2003, AJ, 125, 3046

Dean, J. F., Warren, P. R., \& Cousins, A. W. J. 1978, MNRAS, 183, 569

Della Valle, M. 1988, in The Extragalactic Distance Scale, ed. S. van den Berg, \& C. J. Pritchet (Provo: Brigham Young Univ. Press), APS Conf. Ser., 4, 73

Ferraro, F. R., Messineo, M., Fusi Pecci, F., et al. 1999, AJ, 118, 1738 (F99)

Ferguson, A. M. N., \& Johnson, R. A. 2001, ApJ, 559, L13

Ferguson, A. M. N., Irwin, M. J., Ibata, R. A., et al. 2004, in preparation

Freedman, W. L., Wilson, C. D., \& Madore, F. B. 1991, ApJ, 372, 455

Freedman, W. L., Madore, B. F., Gibson, B. K., et al. 2001, ApJ, 553, 47

Girardi, L., Bressan, A., Bertelli, G., \& Chiosi, C. 2000, A\&AS, 141, 371

Greenhill, L. J., Moran, J. M., Reid, M. J., Menten, K. M., \& Hirabayashi, H. 1993, ApJ, 406, 482

Hippelein, H., Haas, M., Tuffs, R. J., et al. 2003, A\&A, 407, 137

Hubble, E. 1926, ApJ, 63, 236

Kent, S. M. 1987, AJ, 94, 306

Kim, M., Kim, E., Lee, M. G., Sarajedini, A., \& Geisler, D. 2002, AJ, 123,244

Kinman, T. D., Mould, J. R., \& Wood, P. R. 1987, AJ, 93, 833

Laird, J. B., Carney, B. W., Rupen, M. P., \& Latham, D. W. 1988, AJ, 96, 1908

Landolt, A. U. 1992, AJ, 104, 340

Lee, M. G., Freedman, W. L., \& Madore, B. F. 1993, ApJ, 417, 553 (LFM93)

Lee, M. G., Kim, M., Sarajedini, A., Geisler, D., \& Gieren, W. 2002, ApJ, 565, 959

Macri, L. M. 2001, Ph.D. Thesis, Harvard University

Madore, F. B., McAlary, C. W., McLaren, R. A., et al. 1985, ApJ, 294, 560

Madore, B. F., \& Freedman, W. L. 1995, AJ, 109, 1645 (MF95)

Madore, B. F., \& Freedman, W. L. 1998, in Stellar Astrophysics for the Local Group, ed. A. Aparicio, A. Herrero, \& F. Sanchez (Cambridge: Cambridge University Press), 263

Magrini, L., Corradi, R. L. M., Mampaso, A., \& Perinotto, M. 2000, A\&A, 355, 713

McConnachie, A. W., Irwin, M. J., Ferguson, A. M. N., et al. 2004 [arXiv: astro-ph/0401453]

Méndez, B., Davis, M., Moustakas, J., et al. 2002, AJ, 124, 213

Mighell, K. J., \& Rich, R. M. 1995, AJ, 110, 1649

Mould, J., \& Kristian, J. 1986, ApJ, 305, 591

Mould, J. 1987, PASP, 99, 1127

Mould, J., Graham, J. A., Matthews, K., Neugebauer, G., \& Elias, J. 1990, ApJ, 349, 503

Pierce, M. J., Jurcevic, J. S., \& Crabtree, D. 2000, MNRAS, 313, 271

Pritchet, C. J. 1988, in The Extragalactic Distance Scale, ed. S. van den Berg, \& C. J. Pritchet (Provo: Brigham Young Univ. Press), ASP Conf. Ser., 4, 59

Renzini, A., \& Fusi Pecci, F. 1988, ARA\&A, 26, 199

Robin, A. C., Reylé, C., Derrière, S., \& Picaud, S. 2003, A\&A, 409, 523

Rosenberg, A., Aparicio, A., Saviane, I., \& Piotto, G. 2000, A\&AS, 145,451 
Sakai, S., Madore, B. F., \& Freedman, W. L. 1996, ApJ, 461, 713 (SMF96)

Salaris, M., Chieffi, A., \& Straniero, O. 1993, ApJ, 414, 580

Salaris, M., \& Cassisi, S. 1998, MNRAS, 298, 166

Salaris, M., Cassisi, S., \& Weiss, A. 2002, PASP, 114, 375

Sandage, A., \& Carlson, G. 1983, ApJ, 267, L25

Sarajedini, A., Geisler, D., Shommer, R., \& Harding, P. 2000, AJ, 120, 2437

Schechter, P., Matteo, M., \& Saha, A. 1993, PASP, 105, 1342

Schlegel, D. J., Finkbeiner, D. P., Douglas, P., \& Davis, M. 1998, ApJ, 500,525

Schommer, R. A., Christian, C. A., Caldwell, N., Bothun, G. D., \& Huchra, J. 1991, AJ, 101, 873

Stephens, A. W., \& Frogel, J. A. 2002, AJ, 124, 2023
Stetson, P. 2000, PASP, 112, 925

Thompson, I. B., Kaluzny, J., Pych, W., et al. 2001, AJ, 121, 3089

Tiede, G. P., Sarajedini, A., \& Barker, M. K. 2004, AJ, in press [arXiv:astro-ph/0403525] (T04)

Urbaneja, M. A., Herrero, A., Kudritzki, R. P., et al. 2002, A\&A, 386, 1019

Van den Bergh, S. 1991, PASP, 103, 609

Van den Bergh, S. 2000, The Galaxies of the Local Group (Cambridge: Cambridge University Press)

Walker, A. R. 2003, in Stellar Candles for the Extragalactic Distance Scale, ed. D. Alloin, \& W. Gieren (Springer), Lecture Notes in Physics, 635, 265 [arXiv: astro-ph/0303011]

Wilson, C. D., Madore, B. F., \& Freedman, W. L. 1990, AJ, 99, 149

Zinn, R., \& West, M. J. 1984, ApJS, 55, 45 